\title{
Article \\ A Pairing Algorithm for Conflict-Free Crossings of Automated Vehicles at Lightless Intersections
}

\author{
Kimia Chavoshi * ${ }^{*}$, Alexander Genser and Anastasios Kouvelas
}

Citation: Chavoshi, K.; Genser, A.; Kouvelas, A. A Pairing Algorithm for Conflict-Free Crossings of Automated Vehicles at Lightless Intersections. Electronics 2021, 10, 1702.

https://doi.org/10.3390/

electronics10141702

Academic Editors: Yu-Chen Lin and Valentina E. Balas

Received: 23 June 2021

Accepted: 13 July 2021

Published: 16 July 2021

Publisher's Note: MDPI stays neutral with regard to jurisdictional claims in published maps and institutional affiliations.

Copyright: (c) 2021 by the authors. Licensee MDPI, Basel, Switzerland. This article is an open access article distributed under the terms and conditions of the Creative Commons Attribution (CC BY) license (https:// creativecommons.org/licenses/by/ $4.0 /)$.
Department of Civil, Environmental and Geomatic Engineering, Institute for Transport Planning and Systems, ETH Zurich, CH-8093 Zurich, Switzerland; gensera@ethz.ch (A.G.); kouvelas@ethz.ch (A.K.)

* Correspondence: kimiac@ethz.ch; Tel.: +41-44-633-6695

\begin{abstract}
This paper studies the planning of conflict-free and efficient crossings of antagonistic vehicles' movements at lightless intersections. A fully automated infrastructure environment is considered, where all vehicles that enter the intersection area are connected and automated (CAVs), i.e., they are equipped with advanced communication and automation technologies. In such a futuristic environment, traffic lights that regulate the right-of-way of different traffic streams are obsolete because of vehicle communication capabilities. The connectivity is utilized to derive vehicle trajectories such that a safe and efficient crossing of lightless intersections is possible. So far, published studies lack the application to complex intersection layouts. To fill this gap, we introduce a control method for $\mathrm{CAV}$ pairing allowing for the safe, collision-free crossing of the intersecting area and optimize traffic conditions, i.e., total delays of the system. Simulation results demonstrate the feasibility and applicability of the presented approach, given that all the technical specifications (e.g., communications, velocity actuators) are present. Finally, we conduct a sensitivity analysis for the algorithm's main parameters, which provides practical insights for the studied experimental scenarios and other existing algorithms in the literature that tackle this problem.
\end{abstract}

Keywords: connected and automated vehicle (CAV); lightless traffic intersection; conflict-free automated crossings; virtual platoon; pairing algorithm

\section{Introduction}

Transportation has become a fast-moving technical discipline, significantly influenced by novel technologies and methods that allow for tackling today's challenges. One of the driving forces is the increasing demand for mobility in urban areas. High traffic demands need to be served through complex transportation networks, which presses these systems towards their capacity limits. The consequences are problems such as congestion and/or accidents. To address these problems, complex infrastructure elements, such as intersections, are of major interest.

Although signal control systems have evolved over the last decades [1-3], poorly-timed (i.e., not responsive to traffic demand) signal controls cause significant user delays. In addition, the installation of traffic lights is a costly process and therefore needs to be planned carefully.

However, recent technologies such as Vehicle-to-Infrastructure (V2I) and Vehicle-toVehicle (V2V) communications are promising for tackling today's transportation challenges. The deployment of V2I and V2V introduces a fully connected environment allowing to share an extensive set of traffic information and also control automated vehicles. Hence, proposed solutions for optimal route guidance (e.g., [4]) and/or efficient and environmentally friendly motion planning can be utilized, leading to more efficient traffic management and, consequently, mitigation of congestion and elimination of traffic accidents.

Within V2I and V2V, the topic of lightless intersections has gained substantial attention in the last decades. The aforementioned fully connected environment is utilized to derive vehicle trajectories such that a safe and efficient crossing of a non-signalized intersection 
is possible. This objective is accomplished by altering the vehicle dynamics of Connected $\mathrm{AVs}(\mathrm{CAV})$, which ensures that constraints for efficiency, safety, ride comfort are met while traffic lights are obsolete. Several solutions have been proposed for a collision-free movement of CAVs at lightless intersections. Approaches differ in the hierarchical level of control (centralized vs. distributed control), the utilized solution method (e.g., dynamic programming, optimization problems, or classic control theory problems), and the case studies' detail of infrastructure modeling.

Despite the variety of studies on this topic, few of them focus on an intersection layout with more than one lane per leg. Such a set-up provides a higher intersection capacity. Accordingly, there is the opportunity to increase the throughput by developing a proper method to control non-conflicting $\mathrm{CAV}$ movements, allow multiple vehicles to traverse the intersection simultaneously, and exploit capacity. To address this issue, we introduce a pairing algorithm to be utilized along with virtual platooning [5] for a safe and efficient crossing of CAVs at lightless intersections. Every CAV in the system can pair with a non-conflicting approaching vehicle to cross the intersection together. Hence, the pairing algorithm enhances the maximum utilization of capacity. Furthermore, an efficient and safe movement of all CAVs in the system is ensured by solving an online optimization problem.

For the performance assessment of this work, we present two case studies. In the first case study, the algorithm is compared to classical signalized intersection. The case studies' level of detail overcome the over-simplified infrastructure modeling of other studies. The second case study provides a sensitivity analysis with insights about crucial model parameters, such as desired speed, pairing distance, and safety distance. As such algorithms' application to a multiple intersection set-up is still open for research, our results indicate how significant parameters influence the algorithm's behavior. Eventually, the demonstrated simulation results provide insights towards the scaling of our algorithm to networks with multiple intersections.

Finally, we can state the contributions of this paper as follows: (a) the introduction of a novel pairing algorithm combined with virtual platooning that allows controlling more complicated intersection layouts; (b) investigating the methods' performance by comparison with a conventional signal control (c) provide insights to the methods' parameter calibration with an extensive sensitivity analysis.

The remainder of the paper is organized as follows: Section 2 provides an extensive literature review about the recent research on lightless intersections and places the current work into context. Section 3 covers the methodological design of the proposed algorithm, i.e., the experiment assumptions, dynamics of CAVs, algorithmic designs that are utilized for operating a lightless intersection, and the design of the proposed decentralized control problem. Section 4 introduces the set-up of two different case studies, presents the corresponding results and sensitivity analysis, and discusses the findings for future developments of more complex lightless intersections. The paper closes with a conclusion and some thoughts for future work.

\section{Previous Related Works}

Intersections, especially in urban areas, have always been critical elements of transport infrastructure. As several conflicting traffic streams from different approaches connect, the likelihood of an accident increases significantly. The study in [6] shows that around 50\% of all accidents in the United States occur at intersections. One of the traffic engineering methods to improve safety is the installation of traffic lights and a corresponding signal control system. This leads to a safer environment for all present transportation modes (vehicular traffic, public transportation, cyclists, and pedestrians). However, traffic lights introduce certain waiting (lost) times, i.e., delays, and constrain the network's throughput with an upper limit.

One of the most commonly used methodologies for signal control over the last decades is fixed-time signal control. The system operates with a predefined cycle time and corresponding pre-timed red and green phases [7], which usually differ according to the 
time of day. Consequently, no responsiveness to changes in demand for individual intersection approaches is available. To overcome this limitation, semi-actuated and fully actuated/adaptive control systems, such as [8] or [9], have emerged and replaced fixed-time systems in the field. These systems react to a change in vehicle demand (measured with sensors) according to predefined rules (e.g., [10]). Works such as [11] extended adaptive systems with a combination of optimization and a stabilization rule by considering local demand measurements. Hence, the system is reactive to short-time local traffic conditions and minimizes users' waiting time at the intersection [12].

Regardless of how sophisticated the design of a traffic signal control system is, the nature of the problem will not vanish: the installation of traffic lights is costly and can have a negative impact on vehicle delay, vehicle accidents and traffic progression [13]. Nevertheless, technologies such as V2V and V2I open up new possibilities to tackle this limitation. The inter-communication between CAVs and infrastructure allows for optimizing a CAV's arrival time at the intersection, and consequently eliminating conflicting pairs. As a result, the installation of traffic lights at an intersection is obsolete because a control system will ensure a safe and efficient crossing of CAVs. Studies such as [14] state that this can double the utilized capacity of an intersection and introduce a significant reduction in total delays.

Works in the literature that have proposed solutions for this type of problem can be classified as centralized [14-22] and decentralized or distributed traffic control approaches $[5,23-30]$. One of the first works approaching the problem by modeling a Multiagent System (MAS) is proposed by [15]. A reservation system (consisting of an intersection manager and a driver-agent for an approaching vehicle) is compared with a traffic light and an overpass policy. Results show that the introduction of such a connected environment significantly decreases the average system delay and increases throughput. In [31], the authors extend their work by allowing vehicle turns, the ability for vehicles to accelerate while in the intersection ([15] only utilizes constant velocities), and by introducing a detailed definition of the communication protocol between a driver-agent and an intersection manager. Work with global coordination (i.e., centralized approach) is a Model Predictive Control (MPC) scheme to derive optimal vehicle trajectories presented in [16]. The optimal trajectories guarantee (a) a collision-free environment by considering cross-collision points and turning movements, and (b) eliminate unrealistic vehicle dynamics by constraining velocity deviations. Hence, the minimization of the cost function consists of three terms: speed deviation, a risk function (concerning a collision), and the control input, i.e., the acceleration term. Because of the problem's computational complexity, the authors only consider a subset of vehicles utilized for the computation of global coordination. In addition, this methodology is only evaluated on a small toy example with one lane per intersection approach. The authors have extended their work in [17] by considering all vehicles and providing more insights into the model behavior on a similar case study.

The study in [19] tackles the problem by finding optimal vehicle motion solutions. The authors propose an optimal control solution, where the problem is formulated in twostages: first, the vehicles have to find a standard formation before entering the intersection. Secondly, a solution is found to cross the intersection safely. Nevertheless, the method involves the downside that parts of the solution are computed offline beforehand and utilized as a look-up table afterward. Hence, the method loses generality but is usable in real-time applications.

Contrary to the formulation of control problems in [16,17,19], the work in [18] proposes a dynamic programming approach with a stochastic look-ahead technique based on a Monte Carlo tree search. Concerning the objective of maximizing the system's throughput, near-optimal actions (i.e., acceleration rates) are found. Further, [22] utilizes a conflict duration graph-based method inspired by Gantt charts to implement a coordination framework for lightless intersections. A slot-based system is proposed in [14] by a generalization of the queuing theory, which is based on a request system and evaluated with different policies. This paper's results are compared to a fixed-timed signal control system in a relatively 
simple case study, where only one conflict point, i.e., two interfering vehicle trajectories, is presented.

The second category of methodologies for lightless intersections is decentralized approaches. In [26], a decentralized framework is proposed by formulating an optimal control problem that minimizes energy consumption and maximizes network throughput, respectively. A similar objective is utilized by [32]. Moreover, in [29], the authors extend these works by relaxing the constant speed assumption inside an intersection's merging zone. Furthermore, a speed-dependent rear-end safety constraint is incorporated.

A two-layered coordination strategy with MPC is formulated by [27]. The authors first design an upper-level controller to compute an optimal reference velocity for all vehicles. Secondly, the lower-level controller operates on every single vehicle in the system to track its reference velocity. The study is one of the few considering two interconnected intersections. Nevertheless, the case study is simplistic as traffic is only moving one-directional; no turns are considered. The authors of [25] also propose an MPC framework by minimizing the cost function consisting of three terms: the collision risk, acceleration, and deviation from the desired velocity. Their case study is a simulation-based methodology implemented with the simulation software VISSIM. The intersection is modeled with two through lanes and one left turn per approach, respectively. In [30], the authors also design an MPC that aims to minimize the speed and jerk deviations. The authors of [28] tackle the problem by applying Mixed-Integer Nonlinear Programming (MINLP).

Apart from centralized and decentralized approaches, game theory formulations can incorporate the interactions among vehicles in the system. The authors of [33] formulate a game theory problem aiming for maximizing the throughput and minimizing the accidents and traffic congestion. Game theory is utilized explicitly for avoiding accidents with a strategic game. Nevertheless, the paper only considers a game with two players, and the intersection set-up is very simplistic. The authors of [34] approach the lightless intersection topic by addressing the general problem of trajectory planning in conflict areas. The authors developed a bi-level cooperative strategy where the upper-level provides solutions for the right of way of vehicles that do not perform lane changing. The lower level of the bi-level problem regulates the right of way for vehicles changing lanes. The algorithm allows the derivation of optimal vehicle trajectories and ensures a collision-free environment. In addition to these studies, which mainly assume that the penetration rate of CAVs in the system is $100 \%$, works such as [23] or [24] address the problem by relaxing this assumption, i.e., reducing the CAV penetration rate. In addition to the discussed works, the interested reader is referred to [35], a literature review article about intelligent intersection management systems, where lightless control strategies are also covered.

\section{Methodology}

In this work, we propose a decentralized control methodology to manage CAVs in lightless intersection environments. The methodology is designed to exploit the intersection's maximum capacity while ensuring a collision-free environment. The CAV's movement is modeled with the basic kinematics equations. To sketch the methodology, we introduce the pairing concept that is utilized along with a virtual platooning concept proposed by [5] and the Intelligent Driver Model (IDM). Finally, an optimization problem is formulated to regulate the CAV's movement. Before every component of the framework is introduced, we list the assumptions considered:

- CAV penetration rate of $100 \%$; moreover, CAVs have uniform driving specifications (same acceleration, deceleration, and speed range).

- $\quad$ Every CAV can communicate with other CAVs within a radius around the intersection region, i.e., is able to access information such as current speed, position, and approaching lane.

- $\quad$ Lane changing inside the intersection region is prohibited. Hence, it is assumed that every approaching CAV is already in its target lane in advance. 
- When the CAVs reach the intersection, they follow a designed optimal path to perform the left or right turns.

- The infrastructure system is defined as an isolated system (i.e., no external influences such as spillbacks from other intersections can occur).

\subsection{CAV's Kinematics}

For defining the movements of every $\mathrm{CAV} i$, the basic kinematic model is utilized. Model equations are defined as follows:

$$
\begin{gathered}
x_{i}(k+1)=x_{i}(k)+v_{i}(k) T+\frac{1}{2} a_{i}(k) T^{2}, \\
v_{i}(k+1)=v_{i}(k)+a_{i}(k) T,
\end{gathered}
$$

where $x_{i}(k), v_{i}(k)$, and $a_{i}(k)$ represent the position, speed, and acceleration of vehicle $i$ at time step $k$, respectively; $T$ denotes the time discretization. In this model, $x_{i}$ and $v_{i}$ are the system states, while $a_{i}$ is the control signal. At every time step $k$, thanks to output feedback, the system states are transferred to a control block. Inside the controller block, an optimization problem is designed to find the proper control signals $a_{i}(k)$ to satisfy all constraints and optimize the objective function. Finally, this control signal is fed to the model to update the system states at the next time step $k+1$.

\subsection{Traffic Coordination at Lightless Intersection}

This work aims to develop a decentralized control strategy to manage CAVs traffic within a lightless intersection environment. In such a set-up, vehicles themselves are responsible for arranging their order for crossing the intersection (in a distributed way). Communication features of CAVs enable their collaboration on building and updating the crossing order. To formulate this collaboration, we employ the concept of virtual platoon [5]. In this method, CAVs from all approaching lanes are projected onto a one-dimensional, fictitious lane, called a virtual platoon. Figure 1 illustrates an example of the projection of CAVs onto the virtual platoon lane. As long as CAVs do not collide in the virtual platoon, they can safely cross the intersection in their respective lane.

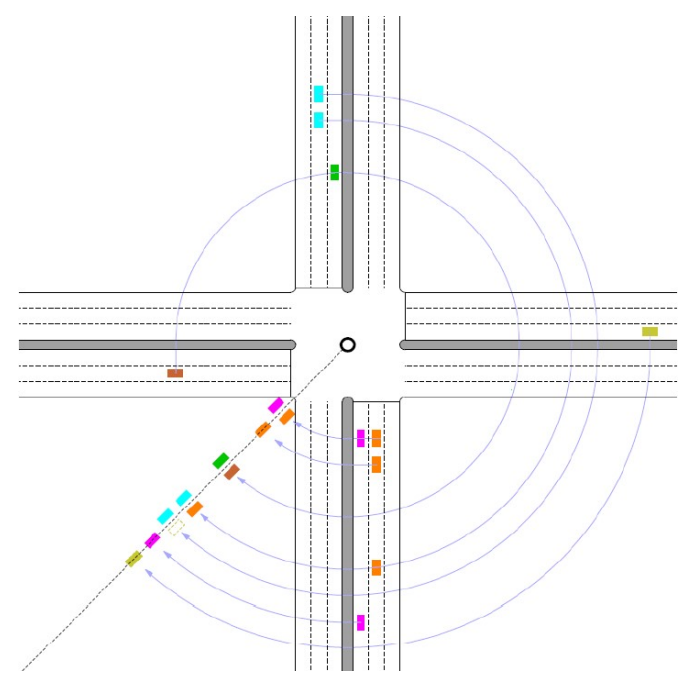

Figure 1. A demonstrative example of a virtual platoon and pairing concepts.

Although the virtual platooning concept can guarantee safety for intersections with one lane per leg, it cannot exploit the maximum available capacity of an intersection with more than one approaching lanes. The potential for a conflict-free and simultaneously efficient crossing of multiple CAVs at the intersection allows the vehicles to arrange the vehicles as a pair in the virtual platoon. Therefore, we introduce the pairing concept in the current paper, which is utilized along with virtual platooning as a lightless strategy. A pair 
is defined as a group of non-conflicting CAVs that are controlled to pass the intersection simultaneously. As soon as a CAV enters the intersection region, it looks for possible pair-mates among other CAVs currently approaching the intersection. Two CAVs can successfully make a pair, if and only if they fulfill the following criteria:

- They must perform non-conflicting movements when crossing the intersection,

- They must be within the pairing radius region, located at the beginning of the intersection approaches,

- Their relative distance must be smaller than or equal to the maximum permitted catch-up distance.

The last two conditions are necessary to ensure the safe formation of pairs before the intersection. In general, the catch-up distance is smaller than or equal to the radius of the pairing region. Once $C A V s$ have identified their pair-mate, they start to catch-up with each other's position and form a pair in the virtual platoon. When the pair is formed, pair-mates continue their movement with the same speed for the intersection region's remaining distance. Figure 1 provides a good visualization and illustration of the application of a virtual platoon and the pairing concept for a four-leg intersection with three approaching lanes per leg. As one can observe, CAVs' locations from all different legs are projected onto the virtual platoon. For instance, Figure 1 shows a demonstrative example with three successfully paired groups, two CAVs that could not manage to find a pair-mate, and one developing pair (the last magenta and blue colored CAVs in the platoon).

To guarantee a collision-free intersection crossing, CAVs are supposed to follow their leader in the virtual platoon (called a virtual leader) with a predefined safety distance $d_{\mathrm{s}, i}$. If the virtual leader is a pair-mate, we set the safety distance to zero; otherwise, we implement the following equation inspired by the Intelligent Driver Model (IDM) proposed by [36]

$$
\begin{gathered}
d_{\mathrm{s}, i}(k)=s_{0}+v_{i}(k) T_{\text {gap }}+d_{i}(k) \\
d_{i}(k)=\max \left(0, \frac{v_{i}(k)\left(v_{i}(k)-v_{\mathrm{VL}, i}(k)\right)}{2 \sqrt{a_{\max } b_{\mathrm{des}}}}\right),
\end{gathered}
$$

where $v_{i}$ and $v_{\mathrm{VL}, i}$ denote the speed of $\mathrm{CAV} i$ (i.e., the follower) and its virtual leader, respectively; $a_{\max }$ denotes the maximum acceleration and $b_{\text {des }}$ the desired deceleration. Note that $b_{\text {des }}$ is smaller than the maximum deceleration to smooth the driving behavior of CAVs. The first term in (3), i.e., $s_{0}$, is the minimum gap between CAVs; the second term expresses the distance that must be kept among CAVs because of the time gap $T_{\text {gap }}$. In the original proposed IDM, $T_{\text {gap }}$ represents the driver's reaction time, but for the CAVs, we replace it with the communication time delay. Finally, the third term, given by (4), is crucial to guarantee collision-free movement, as it indicates the safety distance between CAVs induced by non-zero relative speed.

Algorithm 1 presents the procedure of positioning into the virtual platoon and searching for the pair-mate for every new entered CAV $i$. Via V2V communication, CAV $i$ receives the information of other CAVs (position, origin, destination, and pairing status) inside its communication range. As demonstrated in the following algorithm, the CAV $i$ utilizes this information to check the pairing criteria. Thanks to the access to the current position of other CAVs, CAV $i$ can check if the pairing candidate, $\mathrm{CAV} j$, is within the pairing radius and the relative distance is smaller than catch-up distance. Through origin and destination, it can be assessed whether they belong to a conflict-less set $($ Conflict $(i, j)=0)$. Finally, the pairing status shows if the pairing candidate is not currently engaged in another pair $(\operatorname{Pair}(j)=0)$. If the pairing criteria are fulfilled, CAV $i$ will indicate CAV $j$ as a pair-mate $\left(V L_{i}=j, V L_{\text {type }, i}=\right.$ Pairmate, and $\left.\operatorname{Pair}(i)=1\right)$ as well as send a signal to CAV $j$ to change its pairing status $(\operatorname{Pair}(j)=1)$. In case vehicle $i$ does not manage to find a pair-mate $\left(V L_{i}=\right.$ Nan), it will pick the closest vehicle in the virtual platoon (with the associated minimum relative distance $D(i, k)$ ) as the virtual leader and position itself in the virtual platoon $\left(V L_{i}=k\right.$ and $V L_{\text {type }, i}=$ Leader $)$. 


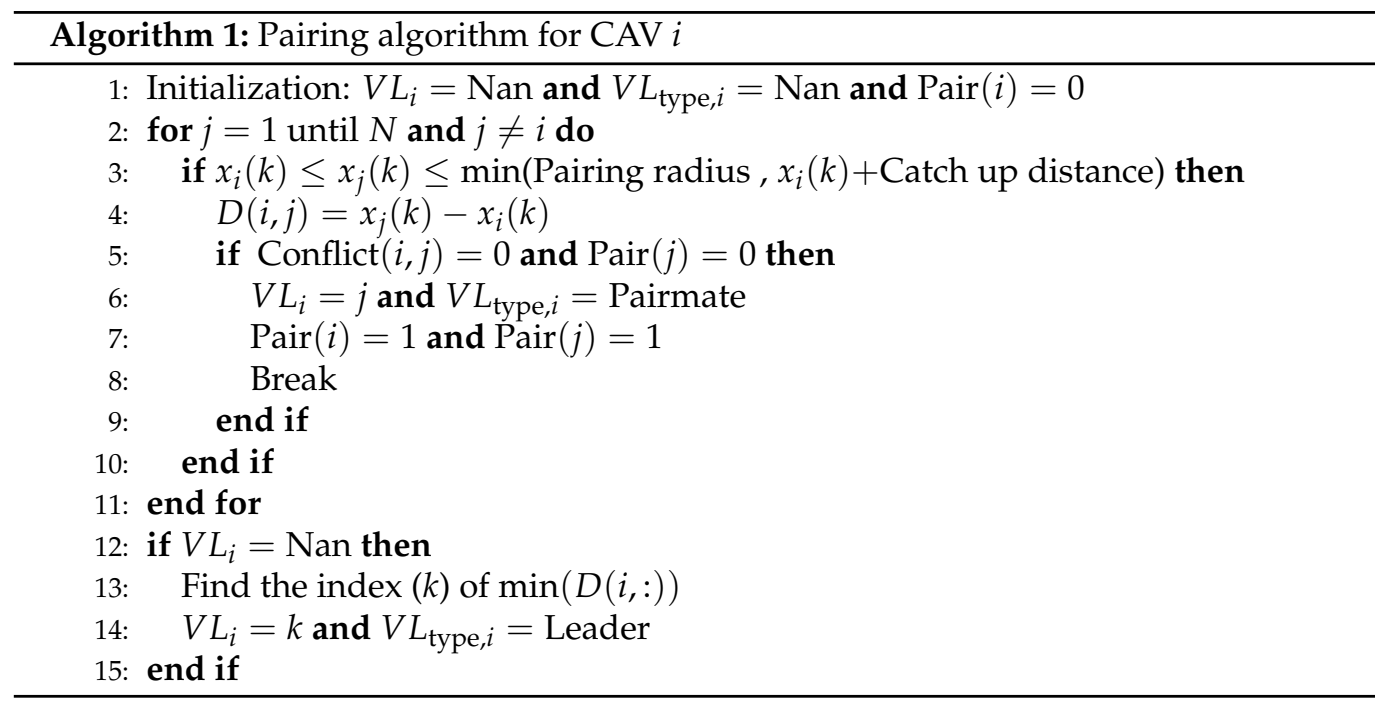

Figure 2 presents a flowchart that summarizes the proposed methodology's implementation on every $\mathrm{CAV}$ and illustrates the resulted procedure for positioning in the virtual platoon. Based on the corresponding traffic demand scenario, new CAVs are generated and assigned to the approaching intersection's legs in every time step. The newly generated CAV will be stored in a queue if there already exists a boundary queue in the relevant approaching leg. Before the CAV can enter the intersection area, it needs to obtain the safety distance (3) with its heading vehicle, the so-called actual leader. This prerequisite is designed to ensure the feasibility of initial conditions. If the CAV does not meet this prerequisite, independently of whether it has already been in the queue or not, it is required to wait in the boundary queue. As already explained, as soon as the CAV enters the intersection area, it is positioned in the virtual platoon. Thanks to Algorithm 1, the recently joined CAV searches for a pair-mate that fulfills the pairing criteria within the pairing region. If the CAV succeeds in finding a pair-mate, it commences forming the pair and catching up with its pair-mate. Otherwise, it follows the virtual leader with a safety distance.

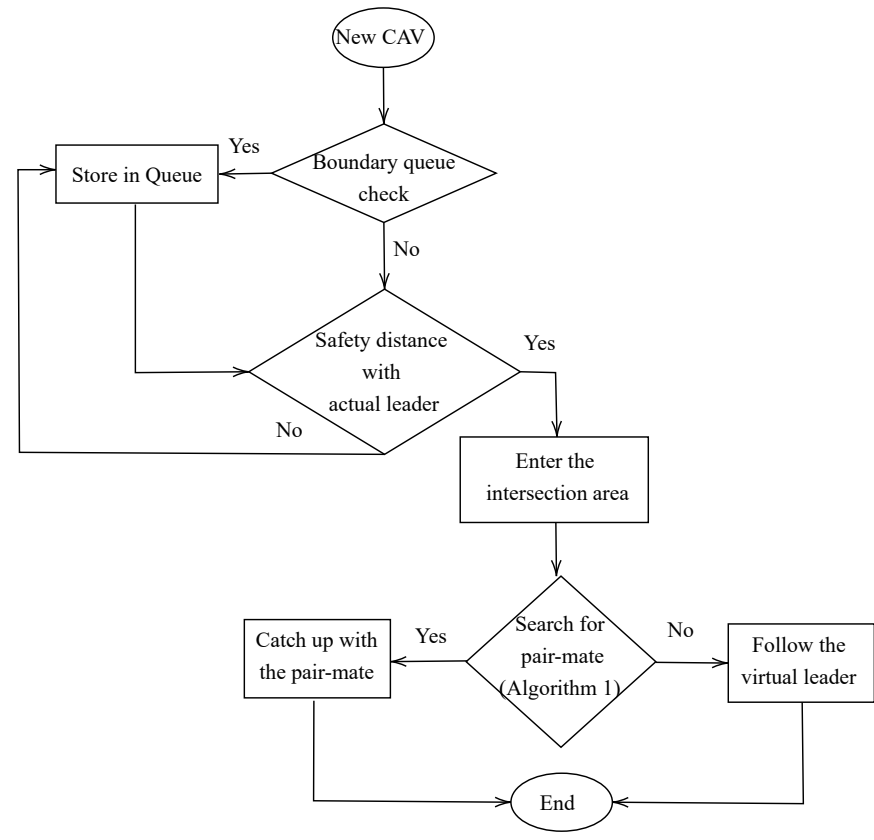

Figure 2. CAV's procedure for positioning in the virtual platoon. 


\subsection{Controller Design}

As discussed in the previous part, the developed pairing algorithm enables every CAV to position itself into the virtual platoon and find a potential pair mate. Every CAV should manage its movement to maintain the desired distance with the virtual leader in the next step. To regulate CAV's movements within the developed lightless intersection area, we formulate the following optimization problem. Solving this optimization problem by every CAV $i$ at time $k$ derives the control input $a_{i}(k)$ required to be applied to the CAV's kinematic model. The distributed optimal regulator is provided by

$$
\begin{gathered}
\underset{a_{i}(k)}{\operatorname{minimize}} f=w_{1}\left(x_{\mathrm{VL}, i}(k)-x_{i}(k)-d_{\mathrm{s}, i}(k)\right)^{2}+ \\
w_{2}\left(v_{i}(k)-v_{\mathrm{des}}\right)^{2}+w_{3} a_{i}(k)^{2}
\end{gathered}
$$

subject to:

$$
\begin{gathered}
a_{\min } \leq a_{i}(k) \leq a_{\max } \\
0 \leq v_{i}(k) \leq v_{\max } \\
x_{\mathrm{AL}, i}(k) \geq x_{i}(k)+s_{0}
\end{gathered}
$$

In this optimization problem, we utilize a quadratic objective function formulation, composed by three terms, where $w_{i}, i \in 1,2,3$, are weights to define each term's proportion. The first term represents the convergence of relative speed with the virtual leader to the safety distance. The second term is designed to keep the deviation between the CAVs speed $v_{i}(k)$ and the desired speed $v_{\text {des }}$ close to zero. Meanwhile, the third term is added to consider the regulator's energy consumption as well as to reduce undesirable control input oscillations.

The objective function is subject to physical constraints: according to (6), acceleration is confined with the maximum deceleration $a_{\min }$ and maximum acceleration $a_{\max }$, respectively; constraint (7) ensures that the speed is always greater or equal than zero and less than the maximum speed $v_{\max }$. To ensure a collision-free movement of all vehicles in the system, we also need to observe the CAVs' movements in the actual lane (i.e., before the intersecting area). Therefore, (8) is included in the optimization constraints to assure that the distance with the actual leader $\left(x_{\mathrm{AL}, i}(k)-x_{i}(k)\right)$ is always greater or equal than the minimum gap $s_{0}$.

Figure 3 sketches the implementation of the proposed distributed methodology in every single CAV. Every CAV $i$, thanks to the V2V communication and pairing concept, identifies and connects to its virtual (VL) and actual leader (AL), respectively. IDM utilizes the received information from leaders and the current states of the CAV to set the safety distance and, accordingly, the reference point. At the next step, the controller will be fed with the current outputs' error in relation to the reference point. We have designed an optimization algorithm (Equations (5)-(8)) for the controller block to address the main objective of the system. The controller will result in a control signal that is the input for the CAV kinematics for the next time step.

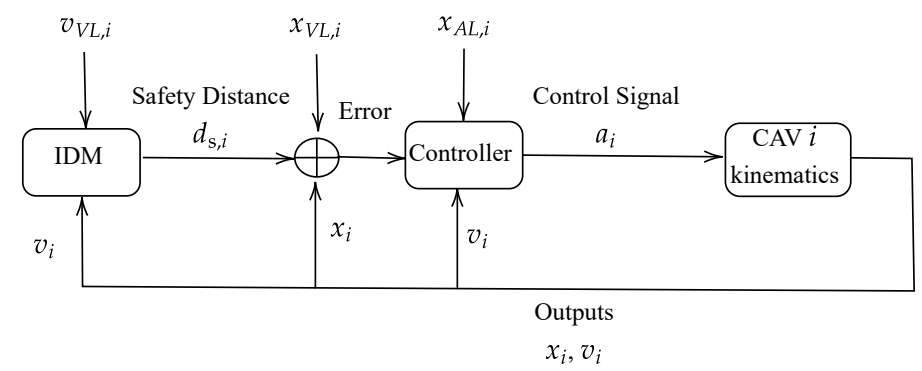

Figure 3. Methodology block diagram. 
Algorithm 2 provides a general overview to the developed methodology.The next section presents simulation results for different scenarios that test the presented distributed controller and evaluate its performance both in terms of safety and traffic efficiency.

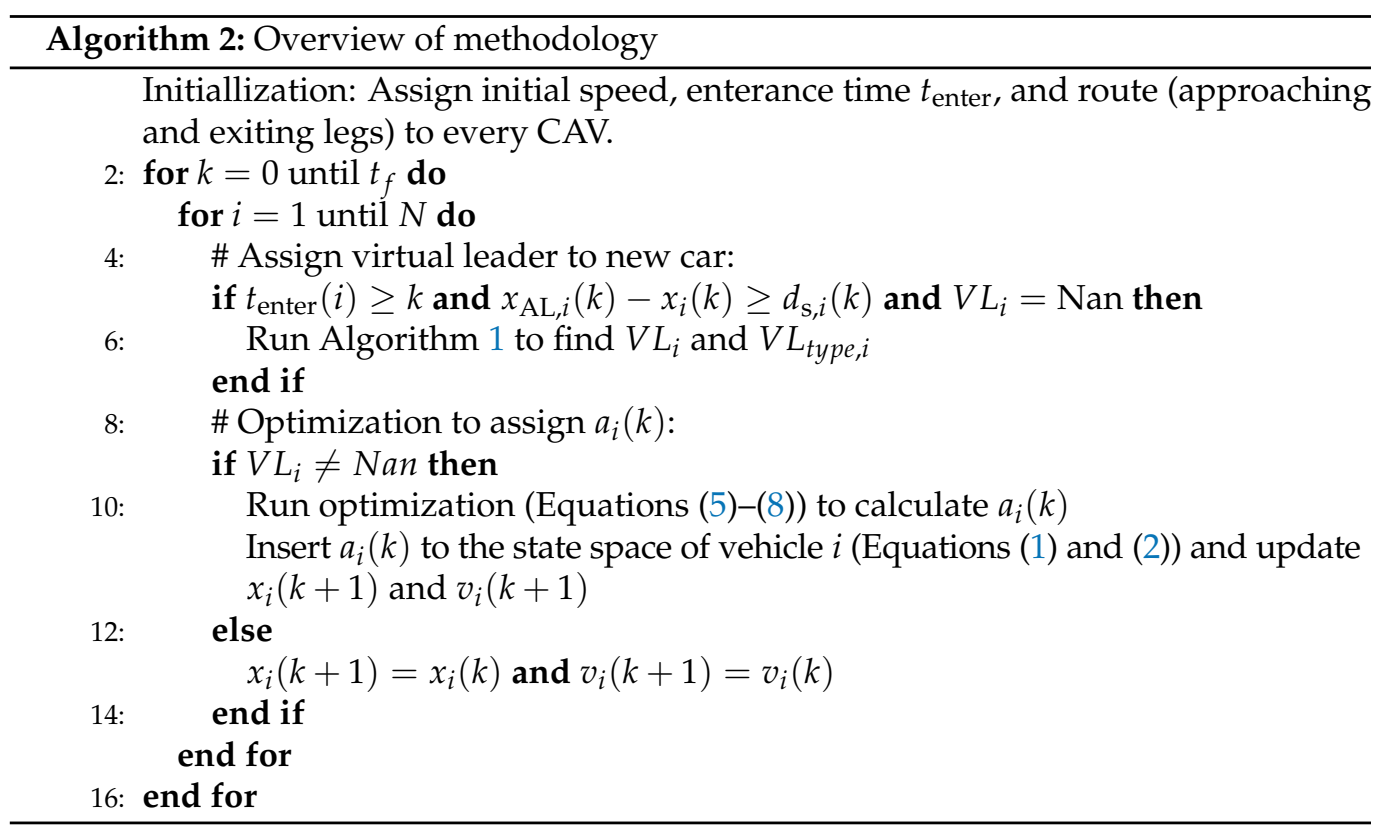

\section{Experimental Simulation Results}

The proposed lightless methodology's performance is analyzed in this section by simulating numerous CAVs movements in two different intersection set-ups. The simulation experiments are executed in MATLAB by developing a code replicating Algorithm 2. The studied system is simulated in discrete time with a time step of $1 \mathrm{~s}$. For vehicle generation, we have used the Poisson distribution to produce random arrivals of CAVs based on a given demand rate $\lambda$. Afterward, generated CAVs are randomly distributed among the approaching intersection's legs.

In the first case study, we apply the proposed lightless methodology to a general intersection layout (Figure 4) and compare the results with a signalized intersection (i.e., a pre-timed signal plan). Through this comparison, we can evaluate the performance of the presented algorithm when compared to classic traffic lights settings. In the second case study, we provide a sensitivity analysis for various parameters of the proposed methodology. We explore the performance in the parameters' space but also test the lightless strategy in a more complex intersection layout (Figure 6).

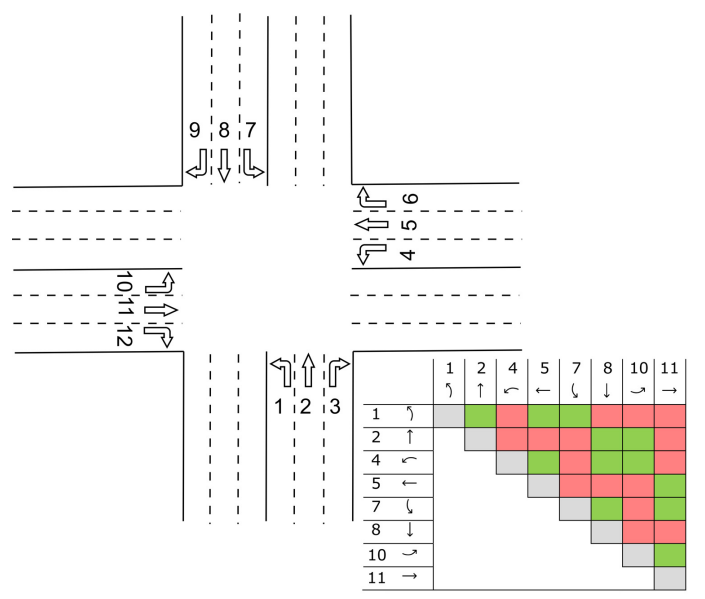

Figure 4. Intersection layout for case study I; non-conflict (green) and conflict (red) sets of movements. 


\subsection{Case Study I}

As the first case study, we consider an intersection with four legs; each leg has three approaching lanes and three departing lanes. Figure 4 demonstrates the general intersection layout for this case study. Every approaching lane corresponds to only one possible maneuver in the intersecting area (right turn, left turn, or through movement). Thereby, each traffic stream is linked to a specific departing lane. This creates a dedicated path for CAVs according to their entering leg and desired destination. Note that the intersecting area is free of lane changing maneuvers. Hence, all CAVs are already placed in their target lane when entering the intersection region.

Note that in the previously described set-up of case study I, the right turns have no conflict with other movements. Therefore, CAVs in such dedicated lanes can freely move through the intersection area. Subsequently, we can exclude the right turns from the set of controlled movements. Figure 4 depicts the remaining paths, grouped as conflicting and non-conflicting movements. Studying the non-conflicting movements reveals that, at most, two non-conflicting CAVs can cross the intersection simultaneously. Hence, the maximum group size of non-conflicting CAVs is two, thus the utilized pairing concept.

The main objective of this case study is to evaluate the presented lightless control strategy in terms of travel delays. Due to this goal, we design two scenarios to manage the CAVs' movement in this case study. In the first scenario, we employ the developed lightless pairing algorithm to control CAVs in the intersection region. Without loss of generality, all lengths of approaching legs are considered $500 \mathrm{~m}$; this creates symmetry in the intersection but this can be easily altered without affecting the results. The pairing region and maximum permitted catch-up distance are considered equal to $75 \mathrm{~m}$. We run this experimental scenario for different desired speeds of $\{7,9,11\} \mathrm{m} / \mathrm{s}$.

For the second scenario, we design a signalized intersection set-up to evaluate the lightless algorithm performance. In this scenario, we implement the IDM for CAVs to follow their actual leader before the intersection zone. Table 1 presents the parameter values that have been used in this scenario. The parameters are evaluated according to the realistic bounds reported by [37]. In the lightless scenario, CAVs are allowed to drive with $v_{\max }$ to catch-up with their leader. Hence, to allow for a fair comparison, we set $v_{\text {des }}=14 \mathrm{~m} / \mathrm{s}$ for the signalized scenario. For our signalized strategy, traffic lights with four phases are modeled at the intersection stopline. Due to the intersection's (and demand) symmetry, for all traffic lights, the green time and red time duration for each phase are set to 20 and $8 \mathrm{~s}$, respectively.

Table 1. The simulation parameters values.

\begin{tabular}{cccccc}
\hline$a_{\max }$ & $a_{\min }$ & $v_{\max }$ & $\boldsymbol{b}_{\text {des }}$ & $\boldsymbol{T}_{\text {gap }}$ & $\boldsymbol{s}_{\mathbf{0}}$ \\
\hline $1.5 \mathrm{~m} / \mathrm{s}^{2}$ & $-3 \mathrm{~m} / \mathrm{s}^{2}$ & $14 \mathrm{~m} / \mathrm{s}$ & $1 \mathrm{~m} / \mathrm{s}^{2}$ & $1.2 \mathrm{~s}$ & $5 \mathrm{~m}$ \\
\hline
\end{tabular}

Both above scenarios are tested with low, medium, and high demands, i.e., $\lambda \in$ $\{1000,2000,3000,4000,5000,6000\} \mathrm{veh} / \mathrm{h}$. In this case study, the simulation time is $4000 \mathrm{~s}$. As the generation of CAVs is performed by sampling from a Poisson distribution, the simulation framework is stochastic. Consequently, for every case 10 simulation repetitions are performed. Figure 5 presents the Average Travel Time (ATT) per CAV for the scenarios of Lightless Intersection (LI) and Signalized Intersection (SI). As demonstrated by the figure's bars, for low demand, i.e., $\lambda \in\{1000,2000\} \mathrm{veh} / \mathrm{h}$, and high demand, i.e., $\lambda \in\{5000,6000\}$ veh/h, LI always achieves better performance for CAVs than the SI scenario. However, for the medium demand, i.e., $\lambda \in\{3000,4000\}$ veh $/ \mathrm{h}$, SI scenario may have better performance if the assigned $v_{\text {des }}$ for the LI case is not sufficiently high (i.e., $11 \mathrm{~m} / \mathrm{s}$ ). Finally, it is worth noting that the LI scenario with $v_{\text {des }}=11 \mathrm{~m} / \mathrm{s}$ outperforms all other scenarios for the entire investigated demand spectrum. 


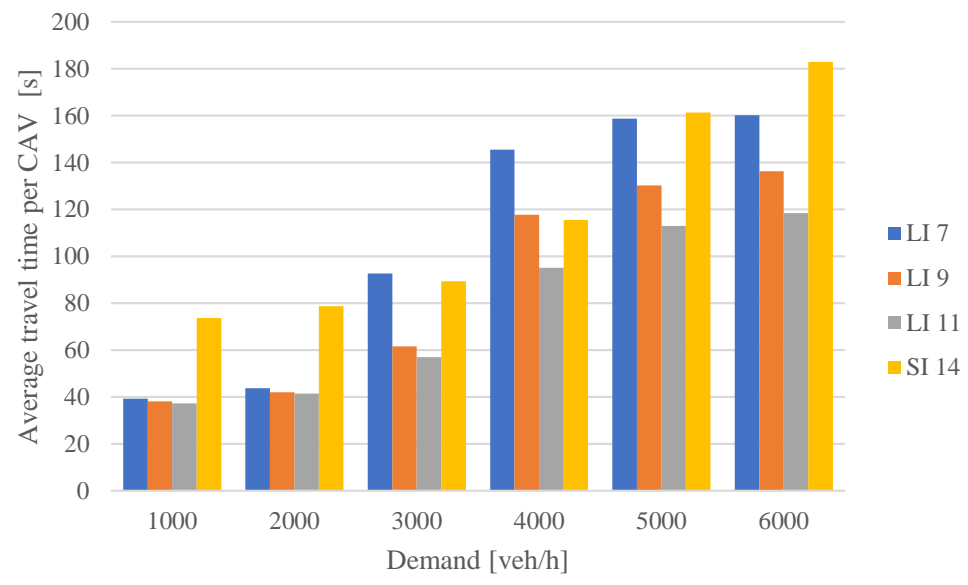

Figure 5. Average travel time per vehicle for Lightless Intersection (LI) and Signalized Intersection (SI) scenarios. The numbers indicate the value of desired speed (in $\mathrm{m} / \mathrm{s}$ ) for every scenario.

\subsection{Case Study II}

As observed previously in the first case study, parameters such as desired speed and demand significantly affect the lightless algorithm's performance. This observation motivates us to analyze the developed algorithm's sensitivity to the different parameters, i.e., demand scenarios, desired speed, safety distance, and pairing region's length. Here, we test the lightless method in a more complex and realistic intersection set-up. The intersection has four legs, where each leg has two approaching lanes and two departing lanes. Figure 6 presents the general intersection layout and compatible paths for this case study. As it can be observed in Figure 6, for every leg, one approaching lane is dedicated to the left turn, while the other lane serves both the through and right turn flows.

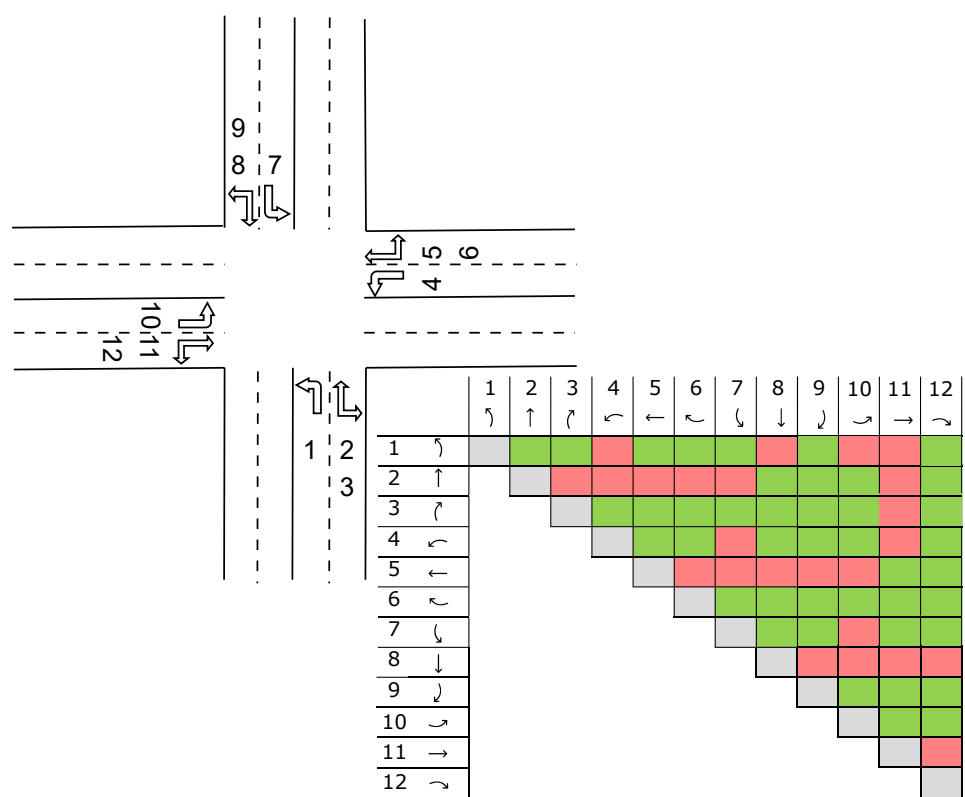

Figure 6. Intersection layout for case study II; non-conflict (green) and conflict (red) sets of movements.

Again, we apply the lightless strategy for CAVs movement in this case study's intersection region. To perform the sensitivity analysis, we utilize the following performance metrics: Vehicle Hours Travelled (VHT), Queue Time, Average Speed, and Total Time Spent (TTS). Since we introduce stochasticity in the simulation (CAV generation), we execute the simulation five times for every assigned demand value. Thereby, it is possible to study the average and standard deviation of all performance metrics. This way, the experiments 
provide more reasonable insights about a realistic performance of the applied strategy. Again, we apply the same parameters values presented in Table 1. In addition, we consider $v_{\text {des }}=11 \mathrm{~m} / \mathrm{s}$ for the lightless methodology.

\subsubsection{Demand Scenarios}

Essentially, pairing is highly dependent on the distribution of the given demand among different approaches. In the case of unevenly distributed demand, the probability of pair formation may drop. To address this issue, we simulate the lightless intersection for two different scenarios, i.e., with different splitting of the total demand among the intersection's movements. In the first scenario, the demand is distributed evenly among the different approaching legs. However, within a leg, the ratio of CAVs moving straight $(52 \%)$ is roughly twice as high compared to the left or right turn ratio $(24 \%)$. In the second scenario, we apply an unevenly distributed demand ratio among the four intersection legs taken from [13].

As we can observe in Figure 7, both splitting scenarios exhibit similar performance patterns for all metrics. As expected, increasing demand results in higher queuing time, lower average speed, and consequently higher VHT. Scenario 1 clearly outperforms scenario 2 in terms of VHT, queuing time, and TTS. However, scenario 2 maintains larger average speeds for medium and high demand. For the rest of the sensitivity analysis, we utilize scenario 1 and demands of $\lambda \in\{1000,3000,5000,7000\} \mathrm{veh} / \mathrm{h}$ in order to cover the spectrum of low, medium, and high demands.
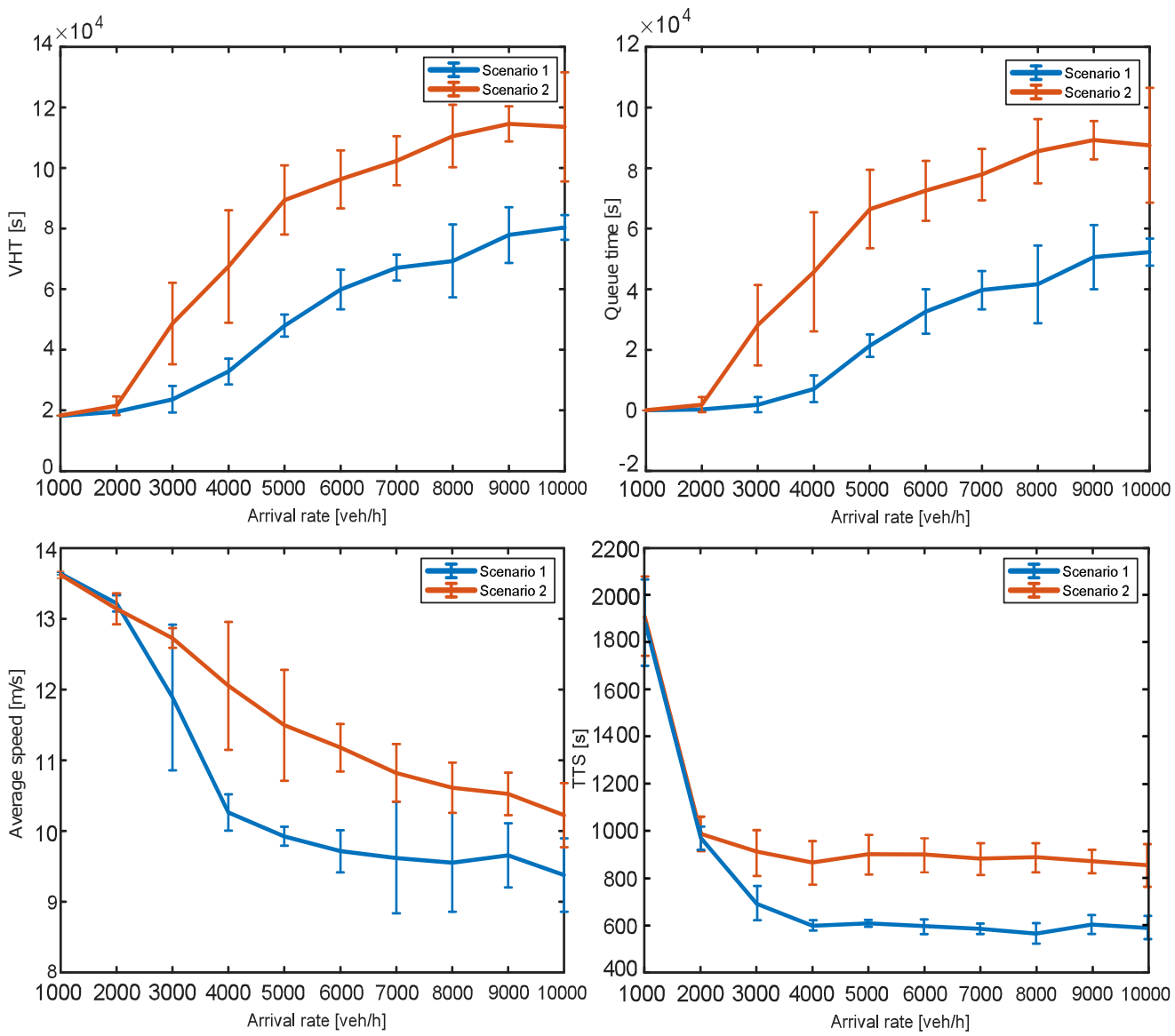

Figure 7. Simulation results for different demand scenarios.

\subsubsection{Desired Speed}

As one can observe in Figure 8, desired speed has contradictory impacts on the methodology. Increasing the desired speed increases the average speed of CAVs in the intersection area. On the other hand, it results in higher queuing times at the controlled 
intersection's zone boundaries. This observation can be explained if we analyze the designed safety policy at the boundaries of the intersection. More precisely, a CAV is allowed to enter the system, provided that it maintains the IDM distance with its actual leader. Looking at Equation (3), increasing the speed will result in a larger IDM safety distance. Hence, CAVs need to wait longer in the boundary queues to let their actual leaders reach the position induced by IDM.
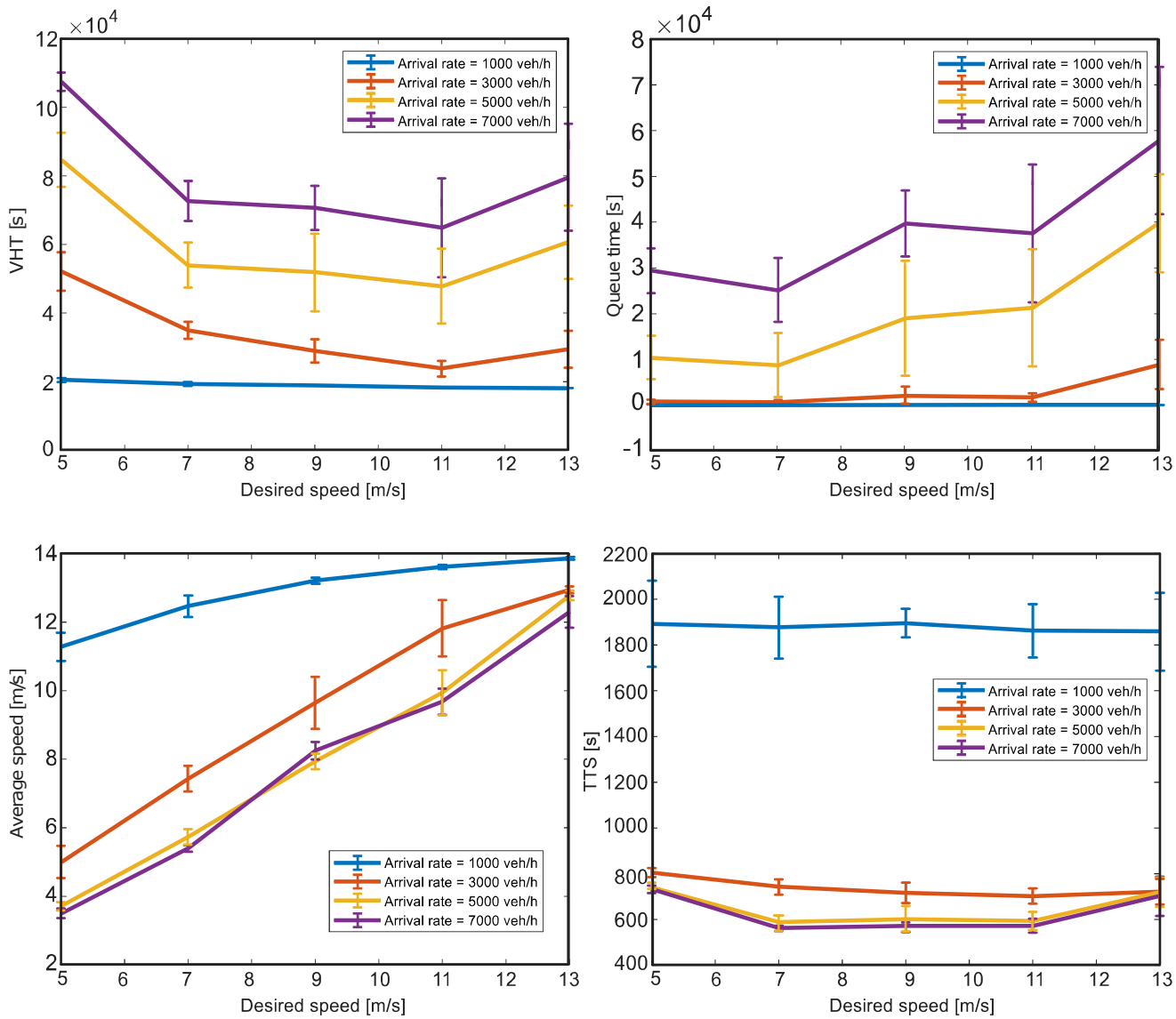

Figure 8. Simulation results for desired speed sensitivity analysis.

Due to these contradictory impacts of desired speed, it is crucial to assign a value that balances this trade-off. VHT is a crucial performance metric for evaluating the proposed algorithm's performance. It refers to queuing time and the time that vehicles spend in the intersection zone, which is a function proportional to average speed. Therefore, we consider VHT as the most proper metric to conduct the speed sensitivity analysis. Figure 8 depicts that the minimum VHT for all demand rates corresponds to the desired speed of $11 \mathrm{~m} / \mathrm{s}$. Furthermore, one can conclude the same result (for desired speed) by observing the TTS scenarios' patterns.

\subsubsection{Safety Distance}

According to Equation (3), safety distance $s_{0}$ is the minimum distance required to be maintained between CAVs. Increasing safety distance increases the IDM distance. Consequently, the queuing time at the boundary of the system increases. As shown in Figure 9, all studied metrics demonstrate the best performance when the lowest safety distance is applied; this is a reasonable result as lower distances increase the system's throughput. 

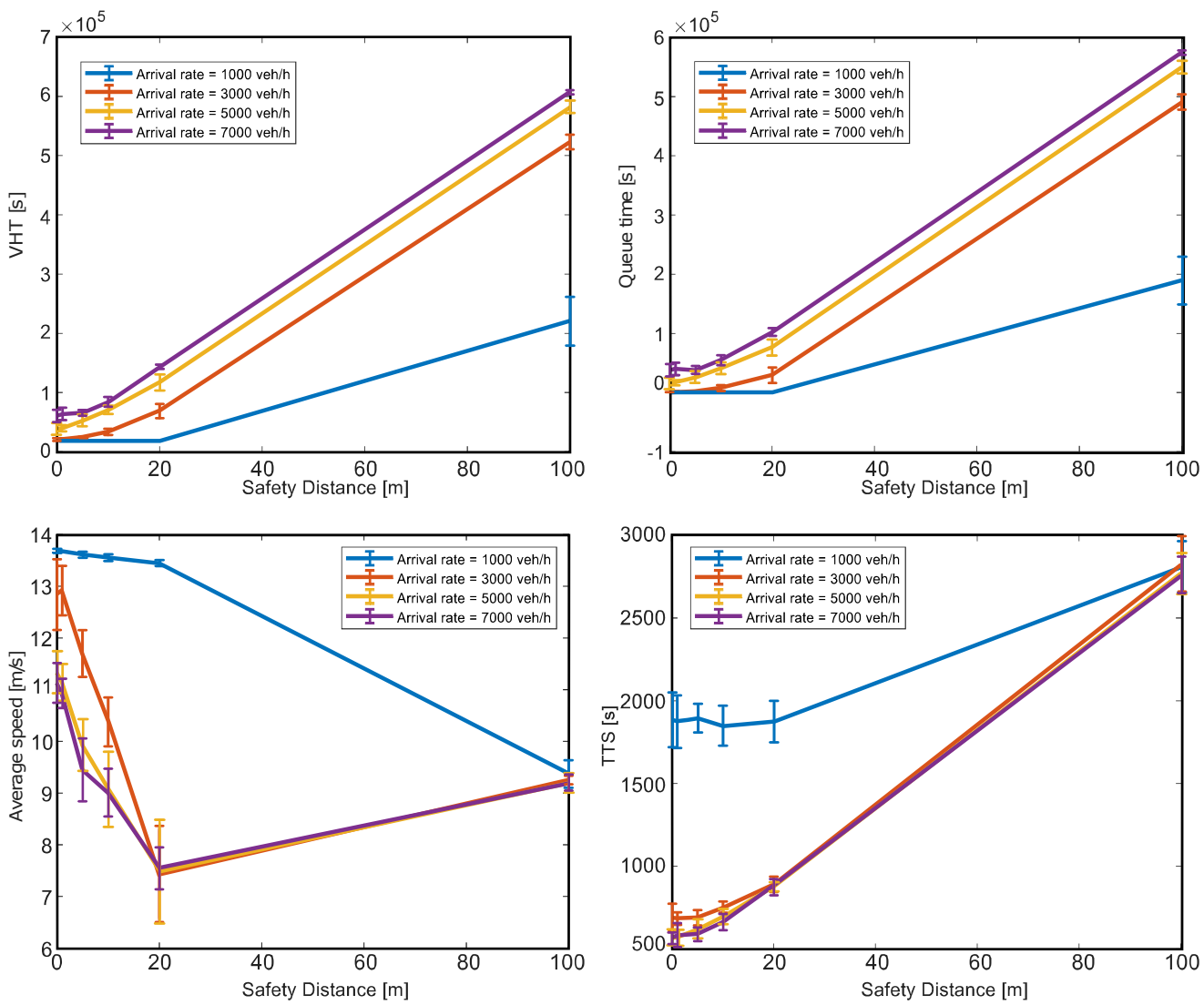

Figure 9. Simulation results for safety distance sensitivity analysis.

\subsubsection{Pairing Region and Catch-Up Distance}

Pairing region and catch-up distance are two essential parameters for the feasibility of the pairing concept. As defined previously, the pairing region is an area located at the beginning of the approaches where a CAV is supposed to look for a pair-mate. Analogously, catch-up distance is defined as the maximum permitted distance between pairing candidates, which ensures they are able to catch up and pair before reaching the cross point. In this part, we assign the same value to both parameters and study the algorithm's sensitivity.

A short pairing region decreases the probability of finding pair-mates and, consequently, the number of pairs; this can result in lower throughput for the intersection. By analyzing the results of Figure 10, we observe that the lowest performance (maximum VHT, queuing time, TTS, and minimum average speed) is obtained for the pairing region of zero (i.e., no pairing is possible). On the other hand, considering a long catch-up distance may result in pairing two vehicles that are relatively far from each other. This phenomenon forces other CAVs located between these pair-mates in the virtual platoon to slow down, and consequently, a shock wave is created; moreover, note that this phenomenon is more likely to occur with lower demand rates. In Figure 10, we can observe the negative effects of these phenomena in terms of simulation average speed. The average speed drops slightly for low and medium demand rates when the pairing distance is set to a value larger than $75 \mathrm{~m}$. 

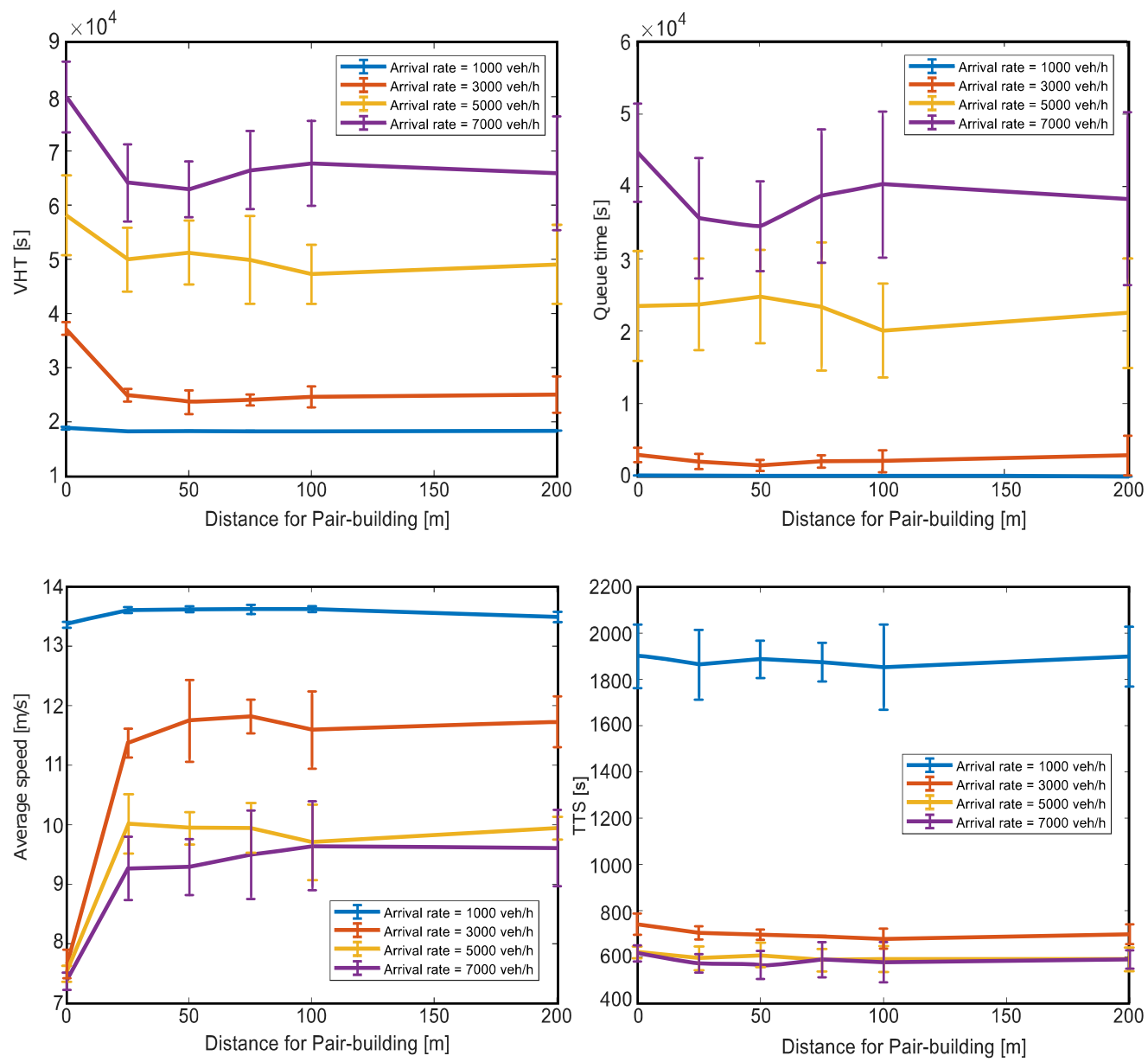

Figure 10. Simulation results for pairing region and catch-up sensitivity analysis.

\section{Conclusions and Future Work}

This paper proposed a novel algorithmic solution for collision-free and efficient crossings of CAVs at lightless intersections. The methodology is simple and utilizes two effective concepts, the projection of all approaching CAVs to a virtual platooning queue and the formation of conflict-free $\mathrm{CAV}$ pairs that can cross the intersection simultaneously. The former is an idea inspired by the literature [5], while the latter is derived by traffic engineering and traffic signal control principles. The pairing concept can increase intersection throughput; however, there is a trade-off between traffic performance and maximum permissible catch-up distance. Simulation experiments and the obtained results have demonstrated the feasibility and applicability of the proposed closed-loop regulator. Note that this is a distributed controller with minimum technical requirements that acts on CAVs' velocity and can improve intersection throughput if tuned properly.

The algorithm has been tested in simulations by utilizing IDM for the kinematic equations. First of all, the results demonstrate that lightless crossings are more efficient than traffic signal plans; this is expected due to the lost times in the intergreen phases. Secondly, the parameters' sensitivity analysis has revealed some interesting findings about desired speed, safety distance, catch-up distance, and the length of pairing distance. More precisely, safety distance is a crucial parameter for the intersection's throughput, and whereas CAV pairing can improve performance, if CAVs have to wait a lot of time for their pair-mate, this can be counterproductive. To conclude, pairing distance is another crucial algorithm parameter, dependent on the demand pattern, that needs to be set accordingly.

Future directions of this research could deal with networks of intersections and coordination of CAVs movements, as well as the surrounding environment (e.g., bicycles, pedestrians) and other (automated) transport modes. Optimal closed-loop feedback solutions is another interesting direction for the controller design. 
Author Contributions: Conceptualization, K.C., A.G. and A.K.; methodology, K.C.; software, K.C. and A.G.; validation, A.G.; formal analysis, K.C., A.G. and A.K.; writing-original draft preparation, K.C. and A.G.; writing-review and editing, A.K.; supervision, A.K. All authors have read and agreed to the published version of the manuscript.

Funding: This research received no external funding.

Acknowledgments: The authors would like to sincerely thank the ETH Zurich students: Josia Meier, Andrea Galli, Tobias Glauser, and Simon Eck for their valuable assistance during the early stages of this project.

Conflicts of Interest: The authors declare no conflict of interest.

\section{References}

1. Diakaki, C.; Papageorgiou, M.; Aboudolas, K. A multivariable regulator approach to traffic-responsive network-wide signal control. Control Eng. Pract. 2002, 10, 183-195. [CrossRef]

2. Kouvelas, A.; Lioris, J.; Fayazi, S.A.; Varaiya, P. Maximum pressure controller for stabilizing queues in signalized arterial networks. Transp. Res. Rec. 2014, 2421, 133-141. [CrossRef]

3. Mercader, P.; Uwayid, W.; Haddad, J. Max-pressure traffic controller based on travel times: An experimental analysis. Transp. Res. Part C Emerg. Technol. 2020, 110, 275-290. [CrossRef]

4. Genser, A.; Kouvelas, A. Optimum route guidance in multi-region networks. A linear approach. In Proceedings of the 99th Annual Meeting of the Transportation Research Board, Washington, DC, USA, 12-16 January 2020.

5. Xu, B.; Li, S.E.; Bian, Y.; Li, S.; Ban, X.J.; Wang, J.; Li, K. Distributed conflict-free cooperation for multiple connected vehicles at unsignalized intersections. Transp. Res. Part C Emerg. Technol. 2018, 93, 322-334. [CrossRef]

6. Golembiewski, G.; Chandler, B.E. Intersection Safety: A Manual for Local Rural Road Owners; FHWA-SA-11-08; Office of Safety Federal Highway Administration: Washington, DC, USA, 2011.

7. Muralidharan, A.; Pedarsani, R.; Varaiya, P. Analysis of fixed-time control. Transp. Res. Part B Methodol. 2015, 73, 81-90. [CrossRef]

8. He, Q.; Head, K.L.; Ding, J. Multi-modal traffic signal control with priority, signal actuation and coordination. Transp. Res. Part C Emerg. Technol. 2014, 46, 65-82. [CrossRef]

9. Pandit, K.; Ghosal, D.; Zhang, H.M.; Chuah, C. Adaptive Traffic Signal Control with Vehicular Ad hoc Networks. IEEE Trans. Veh. Technol. 2013, 62, 1459-1471. [CrossRef]

10. Zaidi, A.A.; Kulcsár, B.; Wymeersch, H. Back-Pressure Traffic Signal Control With Fixed and Adaptive Routing for Urban Vehicular Networks. IEEE Trans. Intell. Transp. Syst. 2016, 17, 2134-2143. [CrossRef]

11. Lämmer, S.; Helbing, D. Self-control of traffic lights and vehicle flows in urban road networks. J. Stat. Mech. Theory Exp. 2008, 2008, P04019. [CrossRef]

12. Genser, A.; Neuenschwander, M.; Kouvelas, A. Wirkungsanalyse Selbst-Steuerung; IVT, ETH: Zurich, Switzerland, 2020.

13. Mannering, F.L.; Washburn, S. Principle of Highway Engineering and Traffic Analysis, 3rd ed.; John Wiley:Hoboken, NJ, USA, 2005.

14. Tachet, R.; Santi, P.; Sobolevsky, S.; Reyes-Castro, L.I. Revisiting Street Intersections Using Slot- Based Systems. PLoS ONE 2016, 11, e014960. [CrossRef]

15. Dresner, K.; Stone, P. Multiagent traffic management: A reservation-based intersection control mechanism. In Proceedings of the Third International Joint Conference on Autonomous Agents and Multiagent Systems, New York, NY, USA, 19-23 July 2004; pp. 530-537.

16. Kamal, M.A.S.; Imura, J.; Ohata, A.; Hayakawa, T.; Aihara, K. Coordination of automated vehicles at a traffic-lightless intersection. In Proceedings of the 16th International IEEE Conference on Intelligent Transportation Systems (ITSC 2013), Hague, The Netherlands, 6-9 October 2013; pp. 922-927.

17. Kamal, M.A.S.; Imura, J.; Hayakawa, T.; Ohata, A.; Aihara, K. A Vehicle-Intersection Coordination Scheme for Smooth Flows of Traffic Without Using Traffic Lights. IEEE Trans. Intell. Transp. Syst. 2015, 16, 1136-1147. [CrossRef]

18. Mirheli, A.; Hajibabai, L.; Hajbabaie, A. Development of a signal-head-free intersection control logic in a fully connected and autonomous vehicle environment. Transp. Res. Part C Emerg. Technol. 2018, 92, 412-425. [CrossRef]

19. Li, B.; Zhang, Y.; Zhang, Y.; Jia, N.; Ge, Y. Near-Optimal Online Motion Planning of Connected and Automated Vehicles at a Signal-Free and Lane-Free Intersection. In Proceedings of the 2018 IEEE Intelligent Vehicles Symposium (IV), Suzhou, China, 26-30 June 2018; pp. 1432-1437. [CrossRef]

20. Bichiou, Y.; Rakha, H.A. Developing an Optimal Intersection Control System for Automated Connected Vehicles. IEEE Trans. Intell. Transp. Syst. 2019, 20, 1908-1916. [CrossRef]

21. Bichiou, Y.; Rakha, H.A. Real-time optimal intersection control system for automated/cooperative vehicles. Int. J. Transp. Sci. Technol. 2019, 8, 1-12. [CrossRef]

22. Deng, Z.; Shi, Y.; Han, Q.; Lv, L.; Shen, W. A Conflict Duration Graph-Based Coordination Method for Connected and Automated Vehicles at Signal-Free Intersections. Appl. Sci. 2020, 10, 6223. [CrossRef] 
23. Yang, B.; Monterola, C. Efficient intersection control for minimally guided vehicles: A self-organised and decentralised approach. Transp. Res. Part C Emerg. Technol. 2016, 72, 283-305. [CrossRef]

24. Yang, B.; Monterola, C. A simple distributed algorithm for lightless intersection control based on non-linear interactions between vehicles. In Proceedings of the 2017 IEEE 20th International Conference on Intelligent Transportation Systems (ITSC), Yokohama, Japan, 16-19 October 2017.

25. Zheng, Y.; Jin, L.; Jiang, Y.; Wang, F.; Guan, X.; Ji, S.; Xu, J. Research on cooperative vehicle intersection control scheme without using traffic lights under the connected vehicles environment. Adv. Mech. Eng. 2017, 9, 1687814017719219. [CrossRef]

26. Malikopoulos, A.A.; Cassandras, C.G.; Zhang, Y.J. A decentralized energy-optimal control framework for connected automated vehicles at signal-free intersections. Automatica 2018, 93, 244-256. [CrossRef]

27. Du, Z.; HomChaudhuri, B.; Pisu, P. Hierarchical distributed coordination strategy of connected and automated vehicles at multiple intersections. J. Intell. Transp. Syst. 2018, 22, 144-158. [CrossRef]

28. Mirheli, A.; Tajalli, M.; Hajibabai, L.; Hajbabaie, A. A consensus-based distributed trajectory control in a signal-free intersection. Transp. Res. Part C Emerg. Technol. 2019, 100, 161-176. [CrossRef]

29. Zhang, Y.; Cassandras, C.G. Joint Time and Energy-Optimal Control of Connected Automated Vehicles at Signal-Free Intersections with Speed-Dependent Safety Guarantees. In Proceedings of the 2019 IEEE 58th Conference on Decision and Control (CDC), Nice, France, 11-13December 2019; pp. 329-334.

30. Kloock, M.; Scheffe, P.; Marquardt, S.; Maczijewski, J.; Alrifaee, B.; Kowalewski, S. Distributed Model Predictive Intersection Control of Multiple Vehicles. In Proceedings of the 2019 IEEE Intelligent Transportation Systems Conference (ITSC), Auckland, New Zealand, 27-30 October 2019; pp. 1735-1740.

31. Dresner, K.; Stone, P. Multiagent Traffic Management: An Improved Intersection Control Mechanism; AAMAS '05; Association for Computing Machinery: New York, NY, USA, 2005; pp. 471-477.

32. Rios-Torres, J.; Malikopoulos, A.A. A Survey on the Coordination of Connected and Automated Vehicles at Intersections and Merging at Highway On-Ramps. IEEE Trans. Intell. Transp. Syst. 2017, 18, 1066-1077. [CrossRef]

33. Wei, H.; Mashayekhy, L.; Papineau, J. Intersection Management for Connected Autonomous Vehicles: A Game Theoretic Framework. In Proceedings of the 2018 21st International Conference on Intelligent Transportation Systems (ITSC), Maui, HI, USA, 4-7 November 2018; pp. 583-588.

34. Xu, H.; Zhang, Y.; Cassandras, C.G.; Li, L.; Feng, S. A bi-level cooperative driving strategy allowing lane changes. Transp. Res. Part C Emerg. Technol. 2020, 120, 102773. [CrossRef]

35. Namazi, E.; Li, J.; Lu, C. Intelligent Intersection Management Systems Considering Autonomous Vehicles: A Systematic Literature Review. IEEE Access 2019, 7, 91946-91965. [CrossRef]

36. Treiber, M.; Hennecke, A.; Helbing, D. Congested Traffic States in Empirical Observations and Microscopic Simulations. Phys. Rev. E 2000, 62, 1805-1824. [CrossRef]

37. Zhu, M.; Wang, X.; Tarko, A.; Fang, S. Modeling car-following behavior on urban expressways in Shanghai: A naturalistic driving study. Transp. Res. Part C Emerg. Technol. 2018, 93, 425-445. [CrossRef] 